\title{
Skeletal Class II Malocclusion Treated with AdvanSync 2 - A Case Report
}

\author{
Namrata Dogra ${ }^{1}$, Archana Jaglan², M. S. Sidhu³ ${ }^{3}$ Seema Grover ${ }^{4}$, Suman ${ }^{5}$ \\ 1, 2, 3, 4, 5 Department of Orthodontics, SGT University, Gurugram, Haryana, India
}

\section{INTRODUCTION}

Treatment of complex malocclusion poses a challenge for the orthodontist because of its multifactorial aetiology. Class II malocclusion is the most frequently encountered and treated malocclusion in orthodontic practice and affects approximately $14.6 \%$ of the North Indian population. ${ }^{1}$ A common reason for Class II malocclusion is mandibular skeletal retrusion which is the most common characteristic, as reported by McNamara. ${ }^{2}$ This can be caused by genetic or hereditary factors. When evaluating treatment options for Class II patients, the extent of the skeletal discrepancy and the skeletal maturity of the patient needs to be considered. Treatment may range from dental compensation including camouflage with extractions to surgical procedures targeted at moving the jaw at fault. In growing patients, growth modification with functional appliances offers an intermediate treatment option.

Functional appliances are basically of two types; Removable and Fixed. Removable functional appliances such as Activator, Bionator, Frankel Function regulator and Twin Block appliance change Class II relationship by the transmission of soft tissue tension to the dentition. Treatment success with these appliances relies heavily on patient compliance. Therefore, in non-compliant patients, fixed Class II correctors in conjunction with fixed orthodontic appliances are the best choice. ${ }^{3}$ Fixed functional appliances generate continuous stimuli for mandibular growth without break and permit better adaptation to functions like mastication, swallowing, speech and respiration. ${ }^{1}$

The Herbst fixed functional appliance has been used routinely for Class II patients and has undergone many design variations over time. ${ }^{4}$ The AdvanSync2 Class II corrector is a recently introduced fixed functional appliance. It has a much smaller size than the conventional Herbst appliances, is easier to place, activate and remove and most importantly, can be used in conjunction with full-arch fixed appliances throughout. ${ }^{3}$ Here we describe a case report of a patient treated with the AdvanSync2 Class II corrector and the findings observed in the sagittal and vertical dimensions.

\section{PRESENTATION OF CASE}

A 14-year-old male patient reported to the Department of Orthodontics with the complaint of irregularly placed teeth and poor facial appearance. The patient did not have any relevant medical history. On extraoral examination, he had a convex profile, obtuse nasolabial angle, deep mentolabial sulcus and potentially competent lips (Figure 1). Mandible was severely retrognathic with SNB reduced to $72^{\circ}$. ANB of $5^{\circ}$ suggested Class II skeletal malocclusion and FMA of $25^{\circ}$ suggested average growth pattern. Intraorally, Class II malocclusion with mild to moderate crowding was seen in the maxillary anterior with buccally erupted canines.

Rotation was present in the upper first premolar of the left and right sides (Figure 2). Increased overjet of $7 \mathrm{~mm}$, and bilateral Class II molar and canine relationship were found. The patient had a positive visual treatment objective (VTO).
Corresponding Author: Dr. Namrata Dogra, Department of Orthodontics, SGT University, Gurugram, Haryana - 122505, India.

E-mail: namrata.dogra@sgtuniversity.org

DOI: $10.14260 / j e m d s / 2021 / 603$

How to Cite This Article:

Dogra N, Jaglan A, Sidhu M. S., et al. Skeletal class II malocclusion treated with AdvanSync 2 - a case report.J Evolution Med Dent Sci 2021;10(34):2951-2953, DOI: $10.14260 / \mathrm{jemds} / 2021 / 603$

Submission 15-04-2021, Peer Review 18-06-2021, Acceptance 26-06-2021, Published 23-08-2021.

Copyright (C) 2021 Namrata Dogra et al. This is an open access article distributed under Creative Commons Attribution License [Attribution 4.0 International (CC BY 4.0)] 

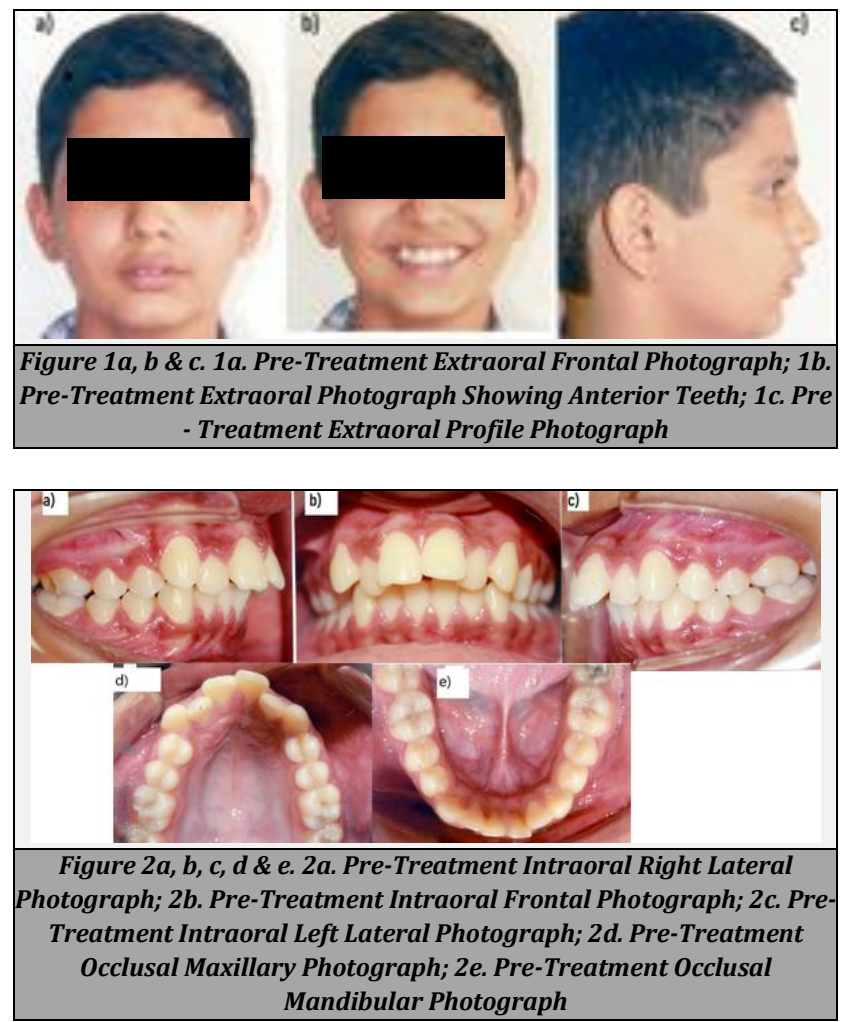

\section{DISCUSSION OF MANAGEMENT}

\section{Treatment 0 bjectives}

1. To improve maxillomandibular Class II skeletal and dental relationship

2. To achieve ideal levelling and alignment of arches

3. To establish ideal overjet and overbite

\section{Treatment Progress}

The treatment plan was discussed with the patient and informed consent was obtained. The treatment plan included a non-extraction treatment with initial levelling and alignment of arches followed by the application of a fixed functional appliance to correct the dental and skeletal sagittal discrepancies. Maxillary and mandibular arches were bonded using .022" × .028" pre-adjusted edgewise MBT appliance prescription. (Di MIM Mini Twin Bracket system, Ortho organizers, A Henry Schein Company). The interproximal reduction was done in the upper anterior and initial levelling and alignment were done in both the arches with $0.14 \mathrm{NiTi}$ wires in the upper and lower arch followed by 0.016 Australian wires and 0.016 x 0.022 S. S wires. An AdvanSync2 Class II corrector (Ormco Corp, Glendora, California) was delivered in the levelling and alignment stage. The AdvanSync2 appliance was reactivated by $2 \mathrm{~mm}$ thrice during the therapy. A Class I skeletal and dental relation was achieved in 11 months (Figure 3). At the end of the functional phase, the arch wires were $19 \times 25 \mathrm{~S}$. S wires in both the arches. After removal of the appliance, $0.016 \times 0.022 \mathrm{~S}$. S wires were placed in upper and lower arches and Class II elastics were started. Post-functional cephalometric analysis revealed an improved ANB angle of $2^{\circ}$ and FMA of $26^{\circ}$, and reduced skeletal convexity (Table 1 ). There was a dramatic improvement in the profile of the patient with the achievement of ideal skeletal and dental relations at the end of the treatment. (Figure 4, Figure 5). Since a majority of tooth movement was completed along with the functional phase, treatment duration also was significantly shortened. The remainder of treatment took an additional 6 months with the patient presented in retention with an upper removable wraparound retainer and a lower canine to a canine lingual bonded retainer. The total treatment time was 17 months.

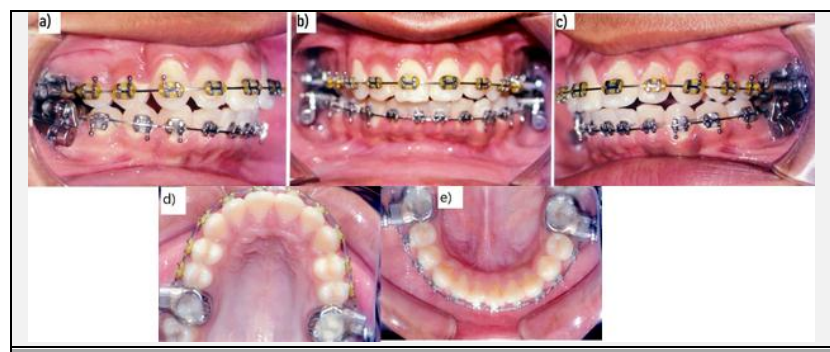

Figure $3 a, b, c, d \&$ e. 3a. Mandibular Advancement with AdvanSync2 3a. Intraoral Right Lateral Photograph; 3b. Intraoral Frontal Photograph; 3c. Intraoral Left Lateral Photograph. 3d. Occlusal Maxillary Photograph; 3e. Occlusal Mandibular Photograph
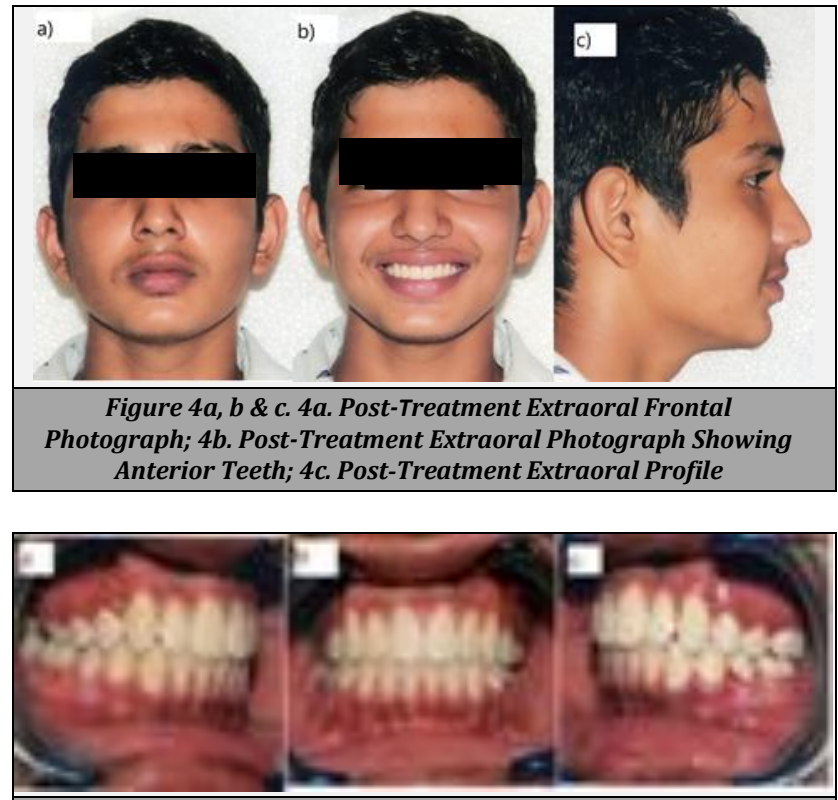

Figure 5a, b \& c. 5a. Post-Treatment Intraoral Right Lateral Photograph; 5b. Post-Treatment Intraoral Frontal Photograph; 5c. Post-Treatment Intraoral Left Lateral Photograph

\begin{tabular}{|ccc|}
\hline Variable & Pre - Treatment & Post - Treatment \\
SNA & 77 degrees & 76 degrees \\
SNB & 72 degrees & 74 degrees \\
ANB & 5 degrees & 2 degrees \\
FMA & 25 degrees & 26 degrees \\
IMPA & 112 degrees & 113 degrees \\
LAFH & $60 \mathrm{~mm}$ & $66 \mathrm{~mm}$ \\
Skeletal convexity & $5 \mathrm{~mm}$ & $2 \mathrm{~mm}$ \\
\hline Table 1. Pre-Treatment and Post-Treatment & Cephalometric Data \\
\hline
\end{tabular}

\section{DISCUSSION}

Class II malocclusion can be treated with different modalities depending upon the growth status of the patient. A growing Class II patient can be effectively managed with functional appliances.

According to Pangrazio et al. removable or fixed functional appliances bring about sagittal and vertical skeletal changes in 
the jaw positions resulting in orthopaedic and orthodontic changes. ${ }^{5}$ Functional appliances induce skeletal growth when used during the active growth periods before or during puberty or Peak Height Velocity.6,7 Functional appliances demand very good compliance from the patient for achieving desirable results.

Non-compliant patients however can be effectively managed by fixed functional appliances. AdvanSync 2 is the recently introduced fixed functional appliance. The advantage of this appliance is that there is no need to align and level the arches before its placement so that the treatment duration is drastically reduced. Thus, reduced treatment time with early sagittal discrepancy correction was associated with better patient motivation and oral hygiene maintenance.8,9 Most of the fixed functional appliances result in increased proclination of lower incisors. ${ }^{10}$ The AdvanSync2 appliance has a unique design of molar-to-molar attachments and is thus expected to reduce lower incisor proclination since there is no attachment in the lower anterior section. But, some lower incisor proclination was present in our findings. This could be because the heavy stainless-steel wires with labial root torque were not in place during the period of mandibular advancement to counteract the anterior vectors of force. According to Ruf and Pancherz, Advansync2 has produced significant mandibular growth changes in postpubertal patients, past their peak height velocities. ${ }^{4}$ This is in accordance with our posttreatment cephalometric findings denoted by increased SNB value as expected with the relocation of the mandible in a sagittal direction. A systematic review and meta-analysis on the stability of cases treated with Class II correctors found that cases treated with the Herbst appliance were the most stable in the long term. ${ }^{11}$ Since the AdvanSync2 appliance is a modification of the original Herbst, results can be expected to be stable.

\section{CONCLUSIONS}

The advansync2 appliance is an effective class II corrector producing significant mandibular advancement and correction of profile. The treatment time is greatly reduced with the application of this appliance.

Financial or other competing interests: None.

Disclosure forms provided by the authors are available with the full text of this article at jemds.com.

\section{REFERENCES}

[1] Kharbanda OP, Sidhu SS, Sundaram KR, et al. Prevalence of malocclusion and its traits in Delhi children. J Indian Orthod Soc 1995;26(3):98-103.

[2] McNamara JA Jr. Components of Class II malocclusion in children 8-10 years of age. Angle Orthod 1981;51(3):177202.

[3] Chitra P, Negi G, Thushar BK, et al. Treatment outcomes in the sagittal and vertical dimensions with the advansync2 Class II corrector-a case series. J Contemp Orthod 2018;2(3):14-26.

[4] Ruf S, Pancherz H. Herbst/ multibracket appliance treatment of Class II Division 1 malocclusions in early and late adulthood: a prospective cephalometric study of consecutively treated subjects. Eur J Orthod 2006;28(4):352-60.

[5] Pangrazio-Kulbersh V, Berger JL, Chermak DS, et al. Treatment effects of the mandibular anterior repositioning appliance on patients with Class II malocclusion. Am J Orthod Dentorfac Orthop 2003;123(3):286-95.

[6] Malmgren 0, Omblus J, Hagg U, et al. Treatment with an orthopaedic appliance system in relation to treatment intensity and growth periods. A study of initial effects. Am J Orthod Dentofacial Orthop 1987;91(2):143-51.

[7] Hagg U, Pancherz H. Dentofacial orthopaedics in relation to chronological age, growth period and skeletal development. An analysis of 72 male patients with Class II division 1 malocclusion treated with the Herbst appliance. Eur J Orthod 1988;10(3):169-76.

[8] Jakobsson SO, Paulin G. The influence of activator treatment on skeletal growth in Angle Class II: 1 cases. A roentgenocephalometric study. Eur J Orthod 1990;12(2):174-84.

[9] Al-Jewair TS, Preston CB, Moll EM, et al. A comparison of the MARA and the AdvanSync functional appliances in the treatment of Class II malocclusion. Angle Orthod 2012;82(5):907-14.

[10] Pancherz H, Anehus-Pancherz M. The headgear effect of the Herbst appliance: a cephalometric long-term study. Am J Orthod Dentofacial Orthop 1993;130(6):510-20.

[11] Bock NC, von Bremen J, Ruf S. Stability of Class II fixed functional appliance therapy-a systematic review and meta-analysis. Eur J Orthod 2016;38(2):129-39. 